\title{
Fat grafting technique for facial rejuvenation: Our experience in Erbil
}

Jalal Hamasalih Fattah*

\section{Abstract}

Background and objective: There is no universal agreement on how one should harvest, process, or inject fat. The objectives of this study were to evaluate our integrated fat grafting technique, reporting our long term result in 189 patients who underwent autologous fat graft for facial rejuvenation.

Methods: The lower abdomen or thigh was chosen as the donor site for fat graft harvesting under low pressure with 10 cc syringe, processed with low speed centrifugation, and then injected into the face using blunt tip $1 \mathrm{~mm}$ cannula in multiple points, using multiple passes, into multiple tissue planes. A total of 189 patients were injected with the above technique for facial rejuvenation. The results were evaluated clinically and photographically and followed for up to five years. Data were analyzed using the statistical package for the social sciences.

Results: Fat absorption leading to volume loss was the commonest minor complication reported in 51 cases (27\%). No major complications were reported in this series. About $70 \%$ of the patients in this series were highly satisfied after first fat grafting. The main cause of dissatisfaction was the volume loss due to fat absorption which necessitated secondary fat grafting.

Conclusion: Our fat grafting technique emphasizes maintaining the viability of transferred fat grafts during their harvest, purification and placement with a good long-term result.

Keywords: Fat graft; Facial rejuvenation; Fat absorption.

\section{Introduction}

Autologous fat grafting has long been considered as a valid option for soft-tissue augmentation in plastic surgery. It can replace the loss of subcutaneous tissue and correct the cosmetic deformities. ${ }^{1,2}$. The technique of autologous fat grafting is composed of a series of procedures and is variable among different surgeons. There is no universal agreement on how one should harvest, process, or inject fat. For example, should manual aspirate or machine suction be used for obtaining fat grafts, centrifugation or only sedimentation, washing or not washing for processing, and how much overcorrection is needed. ${ }^{3,4}$ Many techniques of fat grafting have been described in the literature. ${ }^{5,6}$ It has been a common practice that the donor site of fat grafting is usually selected by the surgeon based on his or her preference or the desired areas chosen by the patient. The question of which is the best donor site to harvest fat grafts remains unclear as most of the previous studies were not conducted scientifically to adequately address this question. ${ }^{7}$. The optimal method for harvest of fat grafts remains controversial. Some surgeons still routinely harvest fat grafts with conventional liposuction. ${ }^{8}$ Most surgeons believe that fat grafts harvested with syringe aspiration or conventional liposuction need to be processed in some way to limit the blood or oil within the lipoaspirate so that only pure fat as a soft-tissue filler will be used for injection. However, this has become a highly controversial issue and currently, there is no agreement among surgeons concerning which is the best method for processing

* Department of Plastic Surgery, College of Medicine, Hawler Medical University, Erbil, Iraq. 
fat grafts. Three primary methods (sedimentation by gravity, filtering technique, and centrifugation) have been used clinically to process fat grafts. ${ }^{9}$ How fat grafts are placed into the recipient site, can be one of the most important steps in fat grafting. An early study by Carpaneda and Ribeiro demonstrate that the ability of fat grafts to obtain nutrition through plasmatic imbibition occurs approximately $0.5 \_1.5 \mathrm{~mm}$ from the edge of the vascularize $\bar{d}$ tissue. ${ }^{10}$ Although the technique of fat grafting has been reported with good success, ${ }^{11,12}$ variable amount of resorption after fat grafting often result in the need for a subsequent procedure of additional fat grafting. This study aimed to evaluate our fat grafting technique, report our long term result in 189 patients who underwent autologous fat graft for facial rejuvenation.

\section{Methods}

Design and sample collection: This is a prospective case series study conducted in Sardam and PAR private hospitals in Erbil city, Kurdistan region-Iraq from June 2009 to June 2015. A total of 193 patients (185 female and eight male) treated with autologous fat graft for facial rejuvenation included in this study. The average age was 40 years $\pm 9.2 \mathrm{SD}$ (from 18 to 62 years). All patients underwent an outpatient procedure and were evaluated clinically and photographically before and after the operation. The indications for autologous fat grafting in this series consisted of temporal, suborbital hollowing, cheek, nasolabial fold, lips, mouth angle, chin and mandibular area.

Exclusion criteria: exclusion criteria include any patient with a hematological disease, chronic liver disease, patients on the anticoagulant, and any patient with a psychological disease. Among the 193 patients, four patients were excluded because of lack of follow up.

\section{Procedure:}

Pre-operative planning is critical when performing fat transplantation. During the pre-operative consultation, medications and allergies are reviewed. The patient is instructed to stop all medications that interfere with the platelet function, two weeks prior to surgery and can restart them one week after the procedure. Appropriate laboratory tests were obtained. The patient is instructed to take $500 \mathrm{mg}$ of Levofloxacin the evening before and continue once daily for three days after the procedure. In cases with a history of previous herpes labialis infection, $400 \mathrm{mg}$ of acyclovir was given on the morning of the procedure and then twice daily for 1 week. Diazepam may be given to any anxious patient, approximately, $1 \mathrm{hr}$ before the procedure. The lower abdomen or thigh in each patient was chosen as the donor site for fat graft harvesting. The tumescent solution containing $0.05 \%$ lidocaine and $1: 1000,000$ of epinephrine are infiltrated into the donor site. If small infiltrate is required, then the procedure carried out under tumescent anesthesia and in larger areas, tumescent anaesthesia with sedation was given. Only in 14 patients, general anesthesia was given. Once the area is infused, it is best to wait $10-15$ min to allow epinephrine to take full effect and for the anaesthesia to infuse evenly through the tissues. A $2 \mathrm{~mm}$ Coleman cannula with one hole in the tip is connected with a $10 \mathrm{ml}$ syringe for fat graft harvesting. An appropriate amount of fat is gently aspirated with finger pressure on the plunger of the syringe without using a suction machine to minimize trauma to the fat grafts. The fat grafts are then washed with normal saline inside the syringe in sterilized condition to remove lidocaine, oil, and residual red blood cells and are then spun at low speed (1500 rpm) for $3 \mathrm{~min}$ in 135 cases. In the remaining 54 cases, the centrifugation speed was 3000 rpm for 3 minutes. After centrifugation, three layers are seen in the syringe (Figure 1), yellow supernatant and blood stained infranatant and the middle layer which primarily consisting of usable fat parcels which are used for fat injection. 
The pure fat transferred into $1 \mathrm{cc}$ syringes to be ready for fat injection. Fat grafts are injected into multiple tissue areas (within subcutaneous tissue and under superficial musculoaponeurotic system) in multiple tunnels and multiple tissue planes, with a $1 \mathrm{cc}$ syringe connecting with a blunt fat injection cannula whose external diameter is $1 \mathrm{~mm}$ (Figure 2).
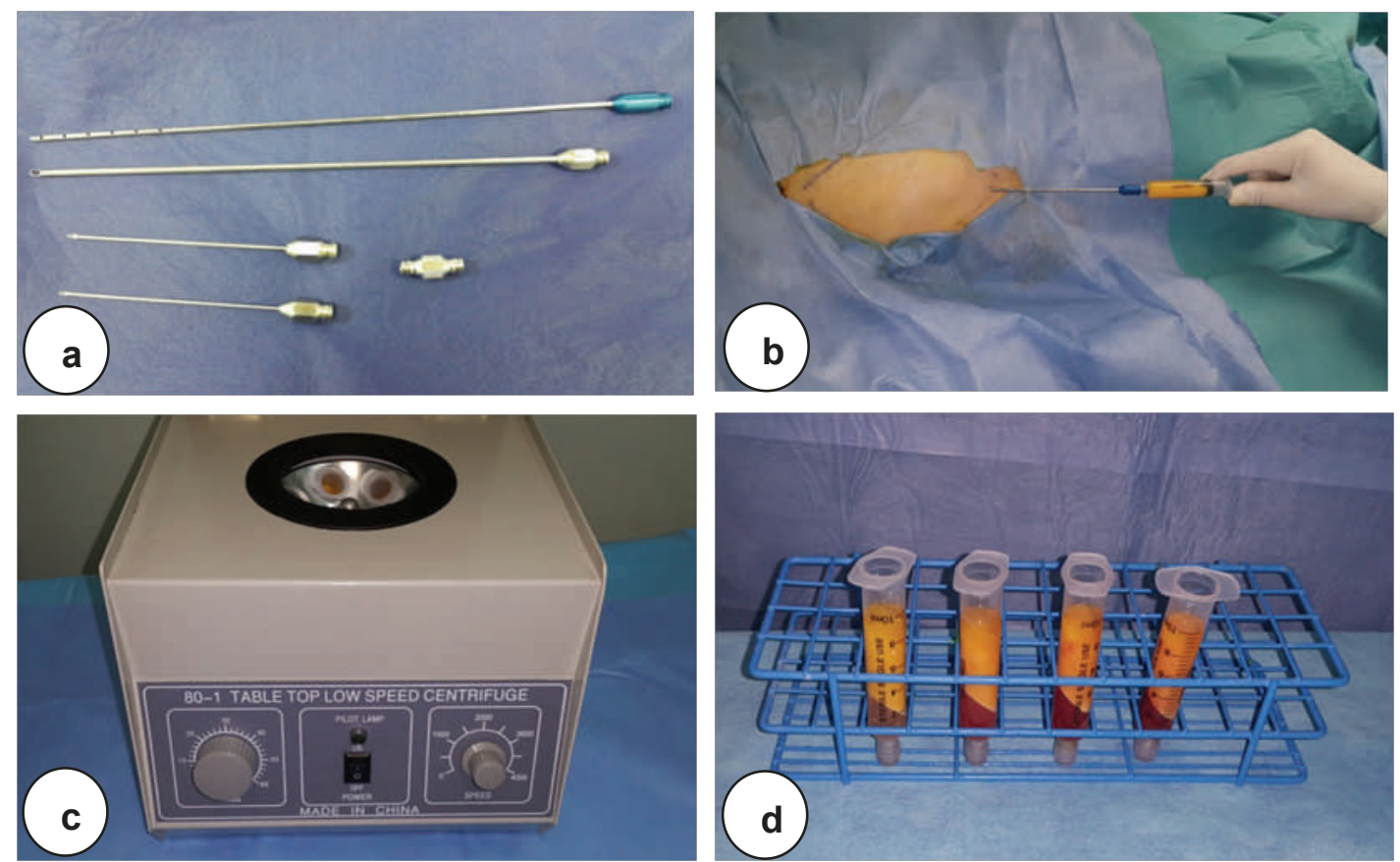

Figure 1: a- Shows Fat harvesting cannula, fat injecting cannula, and connector b- Fat harvesting with $10 \mathrm{ml}$ syringe with finger pressure on the plunger of the syringe c- The fat put in the centrifuge and the speed set at $1500 \mathrm{rpm}$ in 135 cases for three minutes d- After centrifugation, three layers are seen in the syringe, yellow supernatant and blood stained infranatant and the middle layer which primarily consisting of usable fat.
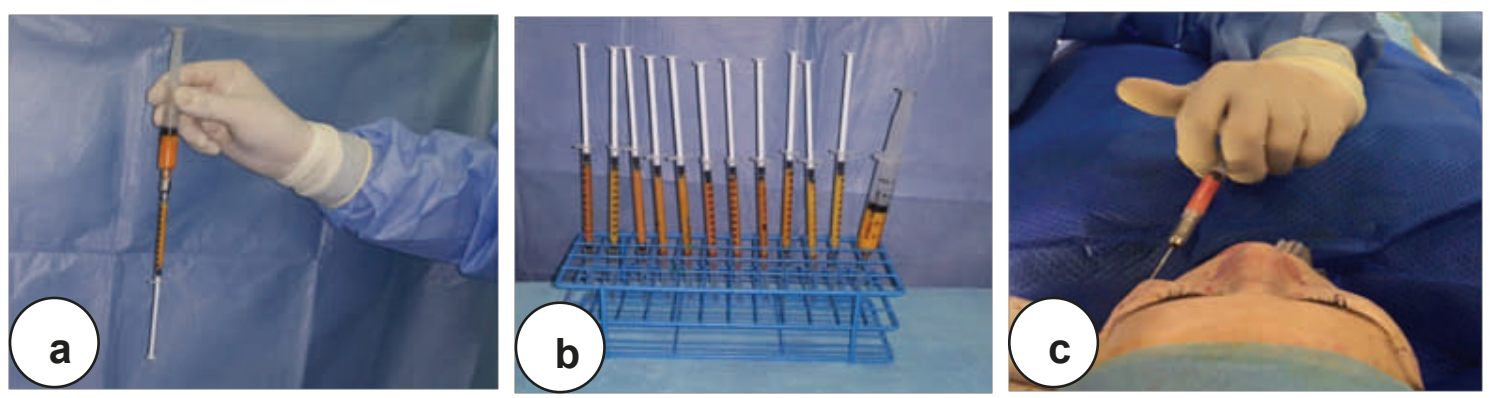

Figure 2: a- The pure fat transferred into $1 \mathrm{cc}$ syringes to be ready for fat injection, b- Fat graft in $1 \mathrm{cc}$ syringes ready for fat injection, c- Fat grafts are injected into multiple tissue areas in multiple tunnels and multiple tissue planes. 
Care is taken to inject fat grafts in only small quantities in one place each time, radially during withdrawal. In the buccal area, the needle should be paralleled to the facial nerve to avoid direct injury to the nerve. The syringe should be drawn back before each injection to check blood return in order to avoid injecting fat grafts into blood vessels causing possible fat emboli. In our practice, we routinely inject about $15 \%$ to $20 \%$ more than required volume of fat grafts to each patient for overcorrection because of possible later absorption after facial recontouring. Gentle massage is done with a finger on the face to ensure a smooth correction. In general, the entire procedure takes about $60-75 \mathrm{~min}$. If necessary, repeated fat grafting can be done after at least six months.

\section{Postoperative care and follow-up}

Patients instructed to take fluid diet and reduce talking to reduce movements of facial muscles in the first week because these movements might traumatize the newly formed blood vessels around injected fat grafts. All patients were seen at postoperative days $7,30,60$ and 120 then yearly and standard photos were taken during each visit. These photos were used to compare the facial with those taken preoperatively. The final results in each patient after operation were evaluated by the patients, and the plastic surgeon according to the clinical improvement, and the comparison of pre- and postoperative photos for the degree of improvement. The degree of improvement was evaluated by both groups and was classified as highly satisfactory, moderately satisfactory, and unsatisfactory. Highly satisfactory i.e. facial contour had been remarkably improved. Moderately satisfactory, i.e., facial contour had been noticeably improved. Unsatisfactory, i.e., facial contour had not been noticeably improved.

Ethical considerations: The study protocol was approved by Medical Ethics Committee of the College of Medicine of Hawler Medical University. Informed consent obtained from all patients.

Statistical analysis: Data were analyzed using the statistical package for social science SPSS V. 19. Chi square test of associates was used to compare between proportions. A $P$ value of less or equal to 0.05 was considered statistically significant.

\section{Results}

The commonest site of fat injection was combined cheek and suborbital area (109 cases, $57.7 \%$ ) as shown in Table 1 . The amount of fat injection is shown in Table 2. Multiple area injections include temporal, suborbital, cheek, mandibular area, nasolabial fold, mouth angle, and chin.

Table 1: Sites of fat injection.

\begin{tabular}{lcc}
\hline Site of fat injection & Frequency & Percent \\
\hline Cheek and suborbital & 109 & 57.7 \\
Cheek & 24 & 12.7 \\
Suborbital & 8 & 4.2 \\
Multiple area & 48 & 25.4 \\
Total & 189 & 100 \\
\hline
\end{tabular}

Table 2: The amount of fat injection.

\begin{tabular}{lcc}
\hline Site of fat injection & Amount of fat injection & $\boldsymbol{P}$ value \\
\hline Cheek and suborbital & $14-22 \mathrm{ml}$ & 0.01 \\
Cheek & $12-20 \mathrm{ml}$ & \\
Suborbital & $2-3 \mathrm{ml}$ & \\
Multiple area & $26-34 \mathrm{ml}$ & \\
\hline \hline
\end{tabular}


Fat grafting technique for facial rejuvenation .......

Zanco J. Med. Sci., Vol. 21, No. (3), December, 2017 https://doi.org/10.15218/zjms.2017.038

Fat absorption was the commonest shown in Table 4. We found that by minor complications (27\%), no major complications were reported as shown in Table 3. Fat injection yields a high satisfaction rate. Nearly $70 \%$ of them were highly satisfied after first fat injection as decreasing the speed of centrifugation from $3000 \mathrm{rpm}$ to $1500 \mathrm{rpm}$ for 3 minutes, there is a significantly lower rate of fat absorption as shown in Table 5.

Table 3: Complications that encountered in this series.

\begin{tabular}{llcc}
\hline & & Frequency & Percent \\
\hline Complications & Fat absorption & 51 & 27 \\
& Oedema & 5 & 2.6 \\
& Echymosis & 3 & 1.6 \\
& Asymmetry & 6 & 3.2 \\
No Complications & & 124 & 65.6 \\
Total & & 189 & 100 \\
\hline
\end{tabular}

Table 4: Satisfaction rate after first fat injection.

\begin{tabular}{lcccc}
\hline & \multicolumn{2}{c}{ Patients' satisfaction } & \multicolumn{2}{c}{ Surgeons' satisfaction } \\
\hline Satisfaction & No. & $\%$ & No. & $\%$ \\
High & 134 & 70.9 & 133 & 70.3 \\
Moderate & 45 & 23.8 & 47 & 24.9 \\
Unsatisfied & 10 & 5.3 & 9 & 4.8 \\
Total & 189 & 100 & 189 & 100 \\
\hline
\end{tabular}

Table 5: The effect of centrifugation speed on fat absorption.

\begin{tabular}{lccccccc}
\hline Fat absorption & \multicolumn{2}{c}{$\mathbf{1 5 0 0} \mathbf{~ r p m}$} & \multicolumn{2}{c}{$\mathbf{3 0 0 0} \mathbf{~ r p m}$} & \multicolumn{2}{c}{ Total } & $\boldsymbol{P}$ value \\
& No. & $\%$ & No. & $\%$ & No. & $\%$ & \\
\hline Yes & 17 & 12.6 & 34 & 63 & 51 & 27 & 0.001 \\
No & 118 & 87.4 & 20 & 37 & 138 & 73 & \\
Total & 135 & 100 & 54 & 100 & 189 & 100 & \\
\hline
\end{tabular}




\section{Discussion}

The efficacy of autologous fat grafting for facial rejuvenation has been debated since its popularization in the 1990s owing to the lack of objective data in the literature regarding longevity, and predictability of the fat grafts. $^{13}$ Despite the lack of objective data, fat grafting has become an increasingly popular technique for facial rejuvenation. The incorporation of fat transfer has been driven by a greater appreciation of the role of volume loss in facial aging and the importance of restoring volume to achieve a natural rejuvenated appearance. $^{14}$ In this series, the commonest site for fat graft injection was to augment suborbital and cheek area simultaneously, which is in agreement with Xie et al. ${ }^{6}$ and Jason et al. ${ }^{13}$ As the midface region tends to be the primary area of age-related volume loss. Clinically, these changes present as tear trough deformities, malar hollowing, and loss of cheek definition. Autologous fat grafting provides a means for addressing all of these signs of aging with a single procedure. The fat grafting is a relatively safe procedure with some minor complications. Fat absorption leading to volume loss was the commonest minor complication reported in 51 cases (27\%) that required secondary fat grafting after six months from the initial procedure, the possibility of the need for secondary fat grafting must be explained for all patients before performing any fat grafting. The volume of fat injected in secondary procedures was considerably less than that used at the original procedure and all performed under local anesthesia. Slight facial edema is inevitable sequelae but it will be resolved within 3-5 days, and this must be explained for the patients, however; significant facial edema was seen in 5 patients which were responded to head elevation, cold sponges, and prednisolone $5 \mathrm{mg}$ tab twice daily for five days. Slight ecchymosis was seen in three patients who resolved within ten days, and mild facial asymmetry observed in 6 patients who may be explained by differences in blood supply or positioning postoperatively like lying on one side at night. They were corrected by touch up fat grafting. Infection were not reported in this series in agreement with Obagi. ${ }^{15}$ who reported only one case of abscess formation following facial fat grating which may be explained by using sterile technique in fat grafting including using sterile centrifuge sleeves, prescribing preoperative and postoperative antibiotics, gentle tissue handling, and placing the fat in tiny quantities in multiple tissue planes. Major complication like blindness was not reported in this series. However; a case of acute blindness was reported by Dreizen ${ }^{16}$ following fat graft to glabellar region due to ocular fat embolism. It is believed that sharp instrumentation and 10-cc syringes with high injection pressures were involved in the majority of cases with a vascular fat embolism. Coleman reports that the use of a blunt-tipped cannula with initial withdrawal prior to injection will decrease the risk of vascular penetration. ${ }^{17}$ To avoid ocular fat embolism we used blunt-tipped cannula with $1 \mathrm{cc}$ syringe with initial withdrawal prior to injection, slow injection of small amount of fat using low injection pressure. The final results evaluated by the patients, and plastic surgeon. About $70 \%$ of the patients in this series were highly satisfied after first fat grafting (Figure 3); However, the remaining $30 \%$ of the patients were either moderately satisfied or unsatisfactory. The main cause of dissatisfaction was the volume loss due to fat absorption between the second and third month post fat grafting which occurred in 51 patients $(27 \%)$ which necessitated secondary fat grafting six month after the first procedure. The volume of fat injected in secondary procedures was considerably less than that used in the original procedure, and all performed under local anesthesia. We found that perioral area has more tendency for fat absorption than other areas of the face. More facial expression and chewing movement may contribute to less fat graft survival. Thus, 
patients should be instructed to avoid unnecessary movement after fat grafting and to slightly overcorrect these particular areas. Almost all of the dissatisfied patients were satisfied after the secondary fat grafting (Figure 4). Our result is consistent with that of other researchers like Coleman, ${ }^{5}$ Ramon et al., ${ }^{18}$ and Kanchwala et al. $^{19}$ who reported the overall take rate of fat grafting ranging from $50-90 \%$. The timing of additional fat grafting should be deferred until six months postoperatively to diminish the inflammatory response. ${ }^{19}$ When we analyzed the rate of fat absorption according to the centrifugation speed. We found that there is a significantly higher rate of fat absorption with a higher speed of centrifugation (out of 54 cases with centrifugation speed of $3000 \mathrm{rpm}$ for 3 minutes, fat absorption occurred in 34 cases, i.e., $63 \%$. While among 135 cases with centrifugation speed of $1500 \mathrm{rpm}$ for 3 minutes in only 17 cases, i.e., $12.6 \%$ fat absorption was observed with P-value of 0.001 which is statistically highly significant). Yoshimura et al. found that centrifugation concentrates adipose tissues and ADSCs (adipose derived stem cells) in the adipose portion. They conclude that excess centrifugation can destroy adipocytes and ADSCs, but appropriate centrifugation concentrates these cells, resulting in enhanced fat graft take. ${ }^{20}$ In the initial 54 cases we put the centrifugation speed at $3000 \mathrm{rpm}$ for 3 minutes according to Coleman recommendation; however, we found significant fat absorption within 2-3 months following fat grafting. By decreasing the centrifugation speed to $1500 \mathrm{rpm}$ for 3 minutes, we found that the rate of fat resorption was significantly less with
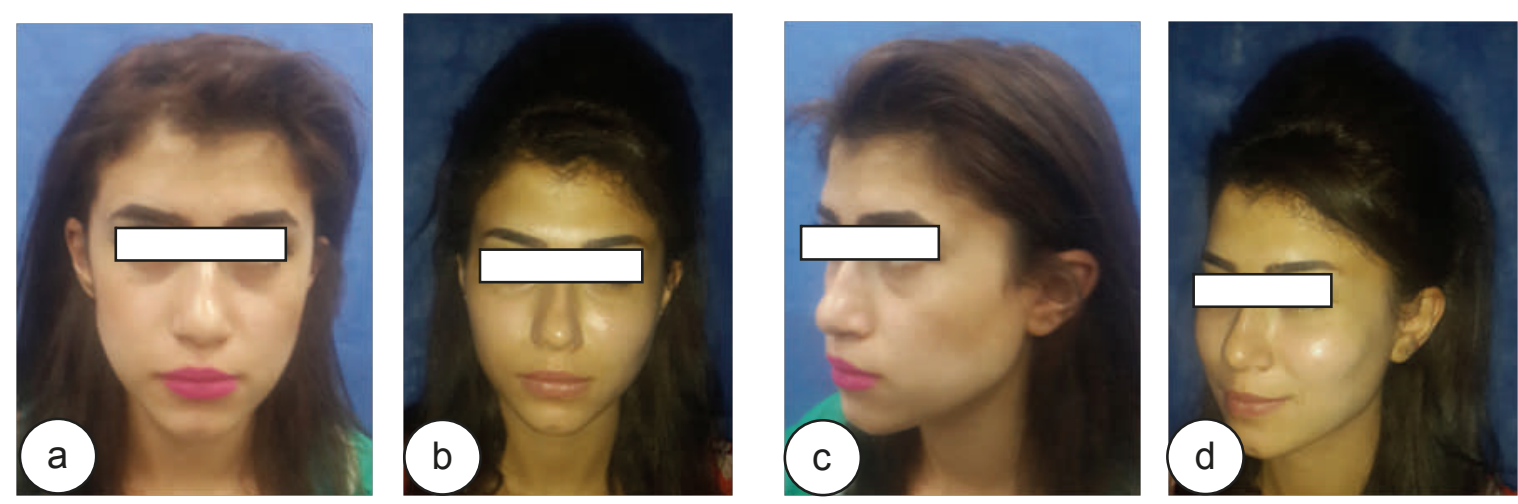

Figure 3: A, C: Before facial fat grafting. B, D: Three years after one session of facial fat grafting shows great improvement of facial appearance and high patient satisfaction.
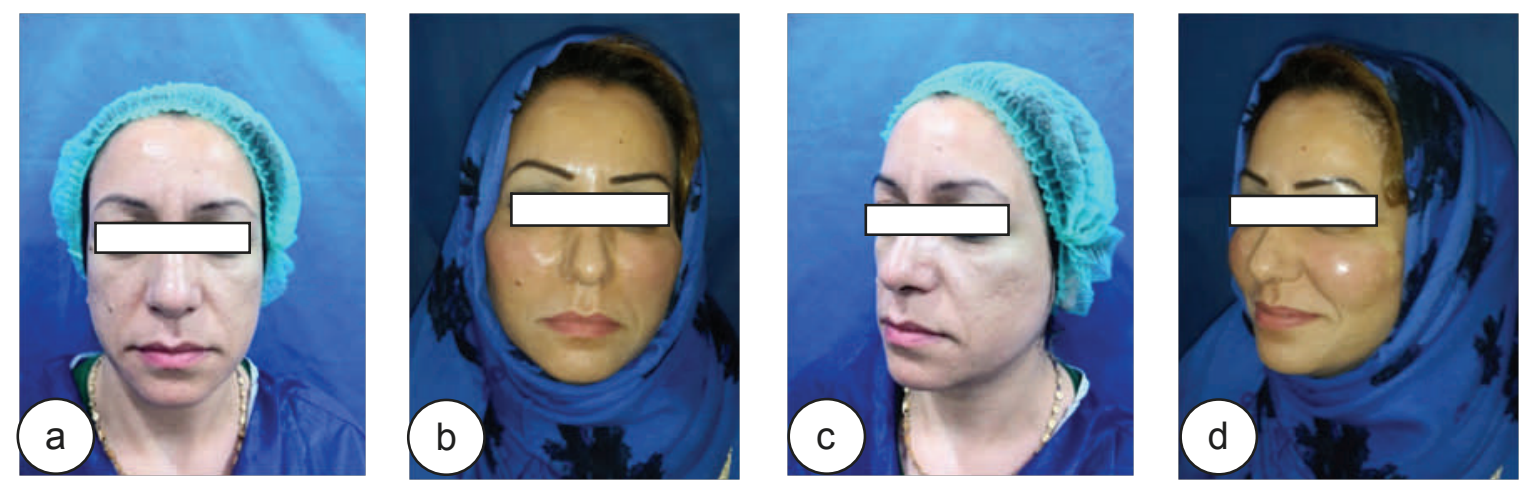

Figure 4: A, C: Before facial fat grafting. B, D: Five years after Two sessions of facial fat grafting shows great improvement of facial appearance and high patient satisfaction. 
consequent increase of patient satisfaction and less need for secondary fat grafting. Our finding is consistent with Xie et al. ${ }^{6}$ and Yoshimura et al. ${ }^{20}$ who recommended low speed centrifugation for better fat survival and take. Coleman refined the technique of fat grafting with the application of centrifugation and a traumatic transfer of fat during operation. This technique was commonly described as the Coleman technique. ${ }^{5}$ Our integrated at grafting technique is similar to Coleman technique with one exception. In Coleman's technique, centrifugation at $3000 \mathrm{rpm}$ for $3 \mathrm{~min}$ is a standard way to process the harvested fat grafts. ${ }^{21}$ However, our study demonstrated that fat grafts had less take and more absorption when the speed of centrifugation is $3000 \mathrm{rpm}$ for 3 minutes. Piasecki et al. with a trypan blue viability assay reported that the reduction of adipocyte viability is directly related to the speed of centrifugation when the speed is higher than $1000 \mathrm{rpm} .{ }^{22}$ Our preferred centrifugation setting (1500 rpm for $3 \mathrm{~min}$ ) can provide not only high purity of fat but also more viable adipocytes within the fat grafts. Our long-term results are encouraging since many of our patients were followed for more than three years with well-maintained transplanted fat.

\section{Conclusion}

According to the present study with up to five years follow up, we believe that autologous fat grafting is a safe and reliable option for mid facial rejuvenation. Our integrated fat grafting technique emphasizes maintaining the viability of transferred fat grafts during their harvest, purification and placement with a good long-term result. However, secondary procedures after six months may be necessary to achieve the desired result.

\section{Conflicts of interest}

The author reports no conflicts of interest.

\section{References}

1. Billings E, May W. Historical review and present status of free fat graft auto transplantation in plastic and reconstructive surgery. Plast Reconstr Surg 1989; 83:368-81.

2. Toledo S, Mauad R. Fat injection: a 20-year revision. Clin Plast Surg 2006; 33:47-53.

3. Kaufman R, Bradley P, Dickinson B. Autologous fattransfer national consensus survey: trends in techniques for harvest, preparation, and application, and perception of short and longterm results. Plast Reconstr Surg 2007; 119:32331.

4. Sinna R, Delay E, Garson S. Scientific bases of fat transfer. Critical review of the literature. Ann Chir Plast Esthet 2006; 51:223-30.

5. Coleman R. Structural fat grafts. Clin Plast Surg 2001; 28:111-9.

6. Xie $\mathrm{Y}$, Zheng $\mathrm{N}$, Li $\mathrm{F}$. An integrated fat grafting technique for cosmetic facial contouring. J Plast Reconstr Aesthetic Surg 2010; 63:270-6.

7. Locke B, Chalain B. Current practice in autologous fat transplantation: suggested clinical guidelines based ona review of recent literature. Ann Plast Surg 2008; 60:98-102.

8. Smith $\mathrm{P}$, Adams $\mathrm{P}$, Lipschitz H. Autologous human fatgrafting: effect of harvesting and preparation techniques onadipocyte graft survival. Plast Reconstr Surg 2006; 117:1836-44.

9. Boschert T, Beckert W, Puckett L, Concannon J. Analysis of lipocyte viability after liposuction. Plast Reconstr Surg 2002; 109:761-7.

10. Carpaneda A, Ribeiro T. Study of the histologic alterations and viability of the adipose graft in humans. Aesthetic Plast Surg 1993; 17:43-7.

11. Kranendonk S, Obagi S. Autologous fat transfer for periorbital rejuvenation: indications, technique, and complications. Dermatol Surg 2007; 33:572-8.

12. Haack J, Friedman O. Facial liposculpture. Facial Plast Surg 2006; 22:147-53.

13. Jason M, Robert G, Mark G. Autologous fat grafting long term evidence of its efficacy in midfacial rejuvenation. Arch Facial Plast Surg 2009; 11(1):24-8.

14. Ersek A. Transplantation of purified autologous fat: a three year follow up is disappointing. Plast Reconstr Surg1991; 87(2):219-27.

15. Obagi S. Autologous fat augmentation and periorbital laser resurfacing complicated by abscess formation. Am J Cosmet Surg 2003; 20:155-7.

16. Dreizen G, Framm L. Sudden unilateral visual loss after autologous fat injection into the glabellar area. Am J Ophthalmol 1989; 107:85-7.

17. Coleman R. Avoidance of arterial occlusion from injection of soft tissue filler. Aesthet Surg J 2002; 22:555-7.

18. Ramon Y, Shoshani O, Peled J. Enhancing the take of injected adipose tissue by a simple method for concentrating fat cells. Plast Reconstr Surg 2005; 115:197-201.

19. Kanchwala K, Glatt S, Conant F, Bucky P. Autologous fat grafting to the reconstructed 
Fat grafting technique for facial rejuvenation .......

Zanco J. Med. Sci., Vol. 21, No. (3), December, 2017

https://doi.org/10.15218/zjms.2017.038

breast: the management of acquired contour deformities. Plast Reconstr Surg 2009; 124: 409-18.

20. Yoshimura K, Sato K, Aoi N, Kurita M, Hirohi T, Harii K. Cell assisted lipostransfer for cosmetic breast augmentation: supportive use of adipose-derived stem/stromal cells. Aesthetic Plast Surg 2008; 32:48-55.

21. Lei H, Li F. Study on influence of centrifugation on fat viability. Chin J Aesthetic Med 2005; 14 : 21-4.

22. Piasecki $H$, Gutowski A, Lahvis $P$. An experimental model for improving fat graft viability and purity. Plast Reconstr Surg 2007; 119:1571-83. 